\title{
The Optimality of Beamforming: A Unified View
}

\author{
Sudhir Srinivasa and Syed Ali Jafar \\ Electrical Engineering and Computer Science \\ University of California Irvine, Irvine, CA 92697-2625 \\ Email:sudhirs@uci.edu,syed@ece.uci.edu
}

\begin{abstract}
We explore the optimality of transmit beamforming for a vector channel with multiple transmit antennas and a single receive antenna. Perfect channel information is assumed to be available at the receiver while the transmitter only has partial/limited knowledge of the user's channel vector based on feedback. Without limiting the kind of partial/limited feedback or the type of channel distribution, we derive a general necessary and sufficient condition for the optimality of beamforming in such channels. The condition we obtain is universal - applicable to all partial/limited feedback scenarios in all ergodic fading channel distributions regardless of the number of transmit antennas or transmit power. Considering different types of partial/limited feedback, we show how our conditions can be employed to obtain previous results on the optimality of beamforming. With Monte Carlo simulations, we provide numerical results comparing different partial/limited feedback schemes.
\end{abstract}

\section{INTRODUCTION}

The fundamental tradeoff in a multiple antenna system between the improvement in system throughput and the amount/quality of channel state information (CSI) at the transmitter (CSIT) and receiver (CSIR) is well documented [1]. While pilot symbols and training techniques can be used to obtain CSI at the receiver, it is impractical to feedback complete and accurate channel information to the transmitter due to the multitude of time-varying channel coefficients. However, even small amounts of channel knowledge at the transmitter can provide significant performance gains compared to systems without any CSIT. This is the stimulus behind the exploration of partial/limited feedback strategies, some of the popular ones being mean/covariance information feedback [2]-[8], magnitude feedback [9], [10] and the more recent quantized direction feedback [11]-[23].

In many of the feedback schemes put forth over the recent years, beamforming is chosen as the transmit strategy. Beamforming is a desirable strategy because it significantly simplifies implementation both at the transmitter and at the receiver. Moreover, it allows for the use of well known scalar codes for the multiple antenna channel by converting the vector channel into a scalar channel. However, in partial CSIT scenarios, subject to the kind and quality of channel information at the transmitter, beamforming may or may not be optimal, i.e., higher rates may be achievable by using more complex strategies. For example, with channel mean information at the transmitter, it has been shown [2]-[4], [24] that when the feedback quality is poor or the SNR is high, capacity is achieved by distributing part of the power along the mean vector and the rest in directions orthogonal to the mean vector. Therefore, in a partial CSIT scenario, the optimality of transmit beamforming determines whether one can obtain the dual benefits of maximum throughput and the low complexity of the beamforming scheme.

Previous work on partial/limited feedback systems [3][5], [17]-[19] has explored the optimality of beamforming for different feedback strategies (magnitude feedback, mean/covariance feedback, quantized direction feedback) in some well known fading channel models. In many of these cases, conditions specifying if and when beamforming is optimal have been provided. Such results are very useful for the specific systems that they consider, however, their scope is limited by the underlying assumptions about the feedback scheme and the channel fade distribution. They do not accommodate any changes to the underlying channel assumptions and/or feedback strategies and are therefore not directly applicable to general partial/limited feedback systems. The main motivation for this work is to provide a unified treatment of the optimality of beamforming for a general partial/limited feedback system. To this end we consider a partial/limited feedback system with multiple transmit antennas and a single receive antenna. We derive a single universal necessary and sufficient condition for the optimality of beamforming (ergodic capacity) that is applicable to all feedback schemes - partial or limited - in all kinds of ergodic fading channel models. We begin with the system model in Section II.

\section{System, Channel and Feedback Model}

Our system model consists of a multiple transmit antenna base station communicating with a single antenna receiver as shown in Figure 1. The complex received signal at the receiver

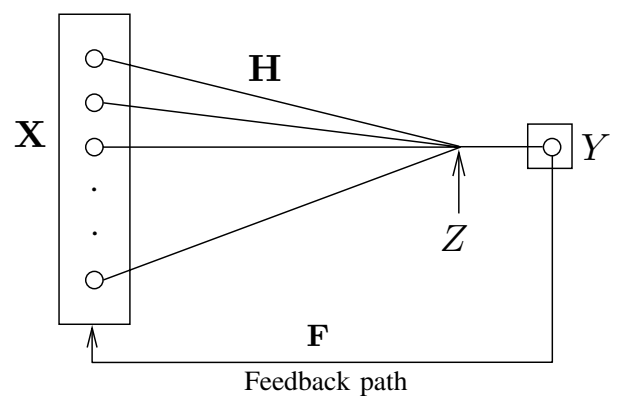

Fig. 1. System Model

is given by the input-output equation:

$$
Y=\langle\mathbf{H}, \mathbf{X}\rangle+Z=\mathbf{H}^{\dagger} \mathbf{X}+Z,
$$


where $\mathbf{X} \in \mathbb{C}^{M}$ is the $(M \times 1)$ transmitted vector of complex symbols, $Y \in \mathbb{C}$ is the received scalar signal, and $Z \sim \mathcal{C N}(0,1)$ is the complex additive white Gaussian noise (AWGN) at the receiver. The time varying narrowband fading channel channel is represented by the $(M \times 1)$ complex vector $\mathbf{H}=\left[\begin{array}{llll}H_{1} & H_{2} & \cdots & H_{M}\end{array}\right]^{T} \in \mathcal{C}^{M}$, where $H_{m}$ denotes the channel gain from the $m^{\text {th }}$ transmit antenna to the receiver. In order to be as exhaustive as possible, we do not restrict $\mathbf{H}$ to any specific distribution.

We assume that there is perfect channel state information at the receiver (CSIR), i.e, the receiver has perfect knowledge of the channel vector $\mathbf{H}$. Based on the kind of scheme, the transmitter obtains partial/limited channel information, usually through a feedback channel.

We denote the feedback information mathematically by the random variable $\mathbf{F}$. The channel feedback $\mathbf{F}$ is a function of the time varying channel state $\mathbf{H}$ or the time varying statistics of $\mathbf{H}$. We define the feedback set $\mathcal{F}$ as the set of all possible realizations of the feedback information $\mathbf{F}$. We now represent some common feedback schemes using these definitions.

1) Perfect feedback [1]: $\mathbf{F}=\mathbf{H}, \mathcal{F}=\mathcal{C}^{M}$.

2) Magnitude Feedback [9], [10]: $f(\mathbf{H})=\|\mathbf{H}\|, \mathcal{F}=\mathcal{R}$.

3) Mean Feedback [2]-[8]: $f(\mathbf{H})=\mathbf{E}_{\mathbf{H}}[\mathbf{H}], \mathcal{F}=\mathcal{C}^{M}$.

4) Covariance Feedback [2]-[8]: $f(\mathbf{H})=\mathrm{E}_{\mathbf{H}}\left[\mathbf{H H}^{\dagger}\right], \mathcal{F}=$ $\left\{\right.$ Positive definite Hermitian matrices in $\mathcal{C}^{M \times M}$ \}.

5) Quantized Feedback [11]-[21]: Described in Section V-D The complex correlation matrix of the input given the feedback $\mathbf{F}$ can be written as $K(\mathbf{F})=\mathrm{E}_{\mathbf{X} \mid \mathbf{F}}\left[\mathbf{X X}^{\dagger}\right]$. The power constraint at the transmitter is given by

$$
\mathrm{E}_{\mathbf{X} \mid \mathbf{F}}\left[\mathbf{X}^{\dagger} \mathbf{X}\right]=\operatorname{Tr}(K(\mathbf{F})) \leq P \quad \mathbf{F} \in \mathcal{F}
$$

The transmit power for all decision regions is the same, equal to $P$. We emphasize here that while this makes the analysis simpler, our results are still applicable even when the transmitter can choose a different transmit power $P_{\mathbf{F}}$ for each feedback $\mathbf{F}$. This simply introduces another optimization problem above the problem statement, where the optimal transmit powers $P_{\mathbf{F}}^{*}$ for feedback $\mathbf{F}$ need to be determined. Ignoring power adaptation is also motivated by several practical considerations as discussed in [19]. We therefore assume the power constraint given by equation (2).

\section{CAPACity EXPRESSION AND PROBlem Statement}

In this section, we consider the capacity optimization problem for the partial/limited feedback system presented in Section II.. The topic of beamforming capacity is discussed leading to the problem statement.

\section{A. Capacity Expression}

The capacity $C_{\text {feedback }}$ of the partial/limited feedback system is given by

$$
C_{f b}=\mathrm{E}_{\mathbf{F}}\left[\max _{\operatorname{Tr}(K(\mathbf{F})) \leq P} \mathrm{E}_{\mathbf{H} \mid \mathbf{F}}\left[\log \left(1+\mathbf{H}^{\dagger} K(\mathbf{F}) \mathbf{H}\right)\right]\right]
$$

The capacity is achieved by transmitting independent complex circular Gaussian symbols along the eigenvectors of $K^{*}(\mathbf{F})$, the optimal input covariance matrix. The powers in the Gaussian symbols are the corresponding eigenvalues of $K^{*}(\mathbf{F})$.

\section{B. Beamforming Capacity}

Suppose we constrain the input covariance matrix to be unit ranked, i.e, fix the transmit strategy to be beamforming. Let the beamforming vector $\mathbf{q}(\mathbf{F})$ be determined from the feedback variable $\mathbf{F} \in \mathcal{F}$. The transmit vector is then given by $\mathbf{X}=\mathbf{q}(\mathbf{F}) x$, where $x$ is a scalar code word symbol and $\mathbf{q}(\mathbf{F})$ is the beamforming vector. To satisfy the transmit power constraint, we set $\|\mathbf{q}(\mathbf{F})\|=1 \quad \forall \mathbf{F} \in \mathcal{F}$ and $\mathrm{E}\left[|x|^{2}\right]=P$. The input covariance matrix will then be $K(\mathbf{F})=P \mathbf{q}(\mathbf{F}) \mathbf{q}^{\dagger}(\mathbf{F})$. The capacity with this additional constraint is called the beamforming capacity and can be expressed as

$$
C_{b f}=\mathrm{E}_{\mathbf{F}}\left[\mathrm{E}_{\mathbf{H} \mid \mathbf{F}}\left[\log \left(1+P \mathbf{H}^{\dagger} \mathbf{q}(\mathbf{F}) \mathbf{q}(\mathbf{F})^{\dagger} \mathbf{H}\right)\right]\right]
$$

With the definitions and expressions obtained above, we now state the problem definition.

\section{Problem Definition}

The problems we take on in this work are the following: For a general partial CSIT model,

- When does beamforming achieve capacity, i.e., when is the optimal input covariance matrix $K^{*}(\mathbf{F})=$ $P \mathbf{q}(\mathbf{F}) \mathbf{q}(\mathbf{F})^{\dagger}$ ?

- What is the optimal beamforming direction, i.e., given $\mathbf{F}$, what is $\mathbf{q}(\mathbf{F})$ ?

\section{OPTIMALITY OF BEAMFORMING}

In this section, we introduce the general conditions for the optimality of beamforming for a partial/limited feedback MISO system with partial/limited feedback. Corresponding conditions for the MIMO case are provided in [25].

\section{A. Universal Conditions for the Optimality of Beamforming}

We provide a mathematical statement of the necessary and sufficient condition for the optimality of beamforming in the following Theorem.

Theorem 1: Beamforming along $\mathbf{q}(\mathbf{F})$ is the optimal transmit strategy if and only if

$$
\beta-\lambda_{\max }[\Lambda] \geq 0,
$$

where $\beta$ and $\Lambda$ are defined as

$$
\begin{aligned}
& \beta=\mathrm{E}_{\mathbf{H} \mid \mathbf{F}}\left[\frac{\left|\mathbf{H}_{\|}\right|^{2}}{1+P\left|\mathbf{H}_{\|}\right|^{2}}\right], \\
& \Lambda=\mathrm{E}_{\mathbf{H} \mid \mathbf{F}}\left[\frac{\mathbf{H H}^{\dagger}}{1+P\left|\mathbf{H}_{\|}\right|^{2}}\right] .
\end{aligned}
$$

$\mathbf{H}_{\|}$is the projection of the channel $\mathbf{H}$ along the beamforming vector $\mathbf{q}(\mathbf{F})$, i.e, $\mathbf{H}_{\|}=\mathbf{H}^{\dagger} \mathbf{q}(\mathbf{F})$. $\lambda_{\max }[\cdot]$ denotes the maximum eigenvalue.

Proof: Proof in [25].

The necessary and sufficient condition presented above is universal - applicable to a wide variety of partial/limited feedback 
strategies regardless of the distribution of the channel, the amount/quality of feedback, the number of transmit antennas or the amount of transmit power.

However, the condition of equation (5) can be split into two simplified conditions that are intuitive and straightforward to work with. We now introduce these conditions.

\section{B. Simplified Universal Conditions}

Let $\mathcal{U}=\left\{\mathbf{u}_{1}=\mathbf{q}(\mathbf{F}), \mathbf{u}_{2}, \cdots, \mathbf{u}_{M-1}, \mathbf{u}_{M}\right\}$ be any set of orthonormal vectors. Let $H_{\perp k}$ be the projection of the channel $\mathbf{H}$ along $\mathbf{u}_{k}$, i.e, $H_{\perp k}=\mathbf{H}^{\dagger} \mathbf{u}_{k}$. Let $\mathbf{H}_{\|}$be the projection of the channel $\mathbf{H}$ along the beamforming vector $\mathbf{q}(\mathbf{F})$, i.e, $\mathbf{H}_{\|}=\mathbf{H}^{\dagger} \mathbf{q}(\mathbf{F})$. Note that $H_{\perp 1}=H_{\|}$. Simplification of the necessary and sufficient condition of Theorem 1 leads to the two conditions listed below:

1) Condition 1:

$$
\mathrm{E}_{\mathbf{H} \mid \mathbf{F}}\left[\frac{H_{\perp k} H_{\perp l}^{*}}{1+P\left|H_{\|}\right|^{2}}\right]=0, \quad 1 \leq k<l \leq M
$$

2) Condition 2:

$$
\mathrm{E}_{\mathbf{H} \mid \mathbf{F}}\left[\frac{\left|H_{\|}\right|^{2}-\left|H_{\perp j}\right|^{2}}{1+P\left|H_{\|}\right|^{2}}\right] \geq 0, \quad 1 \leq j \leq M
$$

Associated with the simplified conditions of equations (9) and (8), we have the following corollaries:

Corollary 1: [Sufficient Condition] Beamforming along $\mathbf{q}(\mathbf{F})$ is the optimal transmit strategy if both Condition 2 and Condition 1 are satisfied.

Corollary 2: [Necessary Condition] Beamforming along $\mathbf{q}(\mathbf{F})$ is the optimal transmit strategy only if Condition 2 is satisfied.

Proof: Proofs for both corollaries in [25].

The corollaries presented above are general in their scope - they are applicable to a variety of partial/limited feedback strategies regardless of the distribution of the channel, the amount/quality of feedback or the number of transmit antennas. Using these corollaries, we now test the optimality of beamforming for different kinds of channel feedback.

\section{Applications}

In the previous section, we have provided universal conditions for the optimality of beamforming for partial/limited feedback systems. In this section we consider some existing feedback strategies and demonstrate that the conditions for the optimality of beamforming in such scenarios can be obtained as special cases of the two conditions of Section IV-B. For each of the feedback scenarios, the corresponding optimal transmit strategies are well known [1], [3], [17], [18]. In order to prove/disprove the optimality of beamforming in these cases, we employ corollaries 1 and 2 and make use of the knowledge of the channel distribution given the feedback to test for conditions (9) and (8).

\section{A. Perfect Feedback}

Recall that for the case of perfect feedback beamforming along the direction of the channel is optimal [1]. From Section II, we have $\mathbf{F}=\mathbf{H}$ and $\mathcal{F} \equiv\{\mathbf{H}\}$. Note that $H_{\|}=\|\mathbf{H}\|$ and $H_{\perp k}=\mathbf{H}^{\dagger} \mathbf{u}_{k}=0$ for $k \geq 2$. Consequently, Condition 2 is always satisfied. Condition 1 reduces to $\frac{\|\mathbf{H}\|^{2}}{1+P\|\mathbf{H}\|^{2}} \geq 0$ which holds for all $\mathbf{H}$. Since both the conditions are satisfied, from corollary 1 , with perfect feedback beamforming is always optimal.

\section{B. Magnitude Feedback}

We assume the magnitude feedback model of [10] in an isotropic fading channel. Mathematically, we can represent the feedback by $\mathbf{F}=\|\mathbf{H}\|$. Since the channel is isotropic and no particular beamforming direction is preferred, any beamforming vector can be chosen to test for optimality. Regardless of the choice of the beamforming vector, the capacity of the system remains the same. For simplicity, we assume that $\mathbf{q}(\mathbf{F})=\left[\begin{array}{llll}1 & 0 & \cdots & 0\end{array}\right]^{\dagger}$. We then have $H_{\perp k}=H_{k}$ and $H_{\|}=H_{1}$. Since $H_{1}$ and $H_{j}(\forall j \geq 2)$ are independent, condition 1 reduces to (assuming $\mathrm{E}\left[\left|H_{i}\right|^{2}\right]=1$ )

$$
\mathrm{E}_{H_{1}}\left[\frac{1}{1+P\left|H_{1}\right|^{2}}\right] \leq \frac{1}{1+P}, \quad 1 \leq j \leq M .
$$

From Jensen's inequality, we have $\mathrm{E}_{H_{1}}\left[\frac{1}{1+P\left|H_{1}\right|^{2}}\right]>\frac{1}{1+P}$, which does not satisfy condition 1 . Since condition 1 is not satisfied, from corollary 2 beamforming is never optimal with magnitude feedback.

\section{No Feedback}

In a no CSIT scenario in isotropic fading, the optimal strategy is not beamforming, but equal power allocation along each of the $M$ dimensions [1]. The no feedback scenario is very similar to the magnitude feedback case, and it can be shown that beamforming is never optimal.

\section{Quantized Direction Feedback}

Quantized direction feedback strategy [11]-[21] is a type of limited feedback scheme where the feedback contains information only about the quantized 'direction' of the user's channel, and no information about the channel 'magnitude'. A predetermined set $\mathcal{Q}=\left\{\mathbf{q}_{1}, \mathbf{q}_{2}, \cdots, \mathbf{q}_{N}\right\}$ of $N=2^{B}$ $(M \times 1)$ unit norm quantization vectors is available to both the transmitter and receiver. The receiver feeds back the index $n^{*}$ of the quantization vector closest to the instantaneous channel, i.e., $n^{*}=\arg \max _{\mathbf{q}_{n} \in \mathcal{Q}}\left|\mathbf{H}^{\dagger} \mathbf{q}_{n}\right|$. The transmitter then uses this quantized channel information to adapt its transmit strategy. We define the 'quantization region' $D_{n}$ for $\mathbf{q}_{n}$ as $D_{n} \triangleq\left\{\mathbf{H}:\left|\mathbf{H}^{\dagger} \mathbf{q}_{n}\right|>\left|\mathbf{H}^{\dagger} \mathbf{q}_{j}\right| \forall j \neq n, 1 \leq j \leq N\right\}$.

In terms of the feedback model of Section II, we have $\mathbf{F}=$ $n^{*}=\arg \max _{\mathbf{q}_{n} \in \mathcal{Q}}\left|\mathbf{H}^{\dagger} \mathbf{q}_{i}\right|$ is the index of the quantization vector $\mathbf{q}_{k}$ closest to the channel $\mathbf{H}$. The feedback set is consequently finite and is given by $\mathcal{F}=\{1,2, \cdots, N\}$.

Without loss of generality we consider the following case:

1) The index sent to the transmitter is $n=1$, i.e., $\mathbf{H} \in D_{1}$. 
2) The co-ordinate system is chosen such that $\mathbf{q}_{1}=$ $\left[\begin{array}{llll}1 & 0 & \cdots & 0\end{array}\right]$.

In such a situation, if $\mathbf{q}(\mathbf{F})=\mathbf{q}_{1}$, it can be seen that $\mathbf{H}_{\|}$, the projection of the channel $\mathbf{H}$ along the beamforming vector $\mathbf{q}$ reduces to $\mathbf{H}_{\|}=\mathbf{H}^{\dagger} \mathbf{q}_{1}=H_{1}$. Similarly we can consider the orthogonal directions to be along the other axes, and $\mathbf{H}_{\perp k}=$ $H_{k}$. The conditions for the optimality of beamforming reduce to:

1) Condition 1 [Symmetry Condition]:

$$
\mathrm{E}_{\mathbf{H} \in D_{1}}\left[\frac{H_{k} H_{l}^{\star}}{1+P\left|H_{1}\right|^{2}}\right]=0, \quad 1 \leq k<l \leq M .
$$

2) Condition 2 [Angular Spread Condition]:

$$
\mathrm{E}_{\mathbf{H} \in D_{1}}\left[\frac{\left|H_{1}\right|^{2}-\left|H_{j}\right|^{2}}{1+P\left|H_{1}\right|^{2}}\right] \geq 0, \quad 1 \leq j \leq M .
$$

Using the conditions, [19] presents several quantized feedback scenarios of interest where beamforming is optimal.

\section{E. Mean Feedback}

In the mean feedback scheme, the transmitter has partial knowledge of the channel distribution, in the form of the mean of the channel vector [2], [4]. The channel, given the feedback, is assumed to be to be white with a known magnitude, i.e., $\mathbf{H} \sim(\mu, \alpha \mathbf{I})$. This kind of channel distribution can be thought of as a mean vector $\mu$ superimposed on an isotropic zero mean Gaussian vector. The results in [4] for the multiple transmit antenna channel with multiple users mimic the results for the MISO case in [2] - the optimality of beamforming depends on the quality of the feedback.

For the mean feedback strategy [2], the instantaneous value of the feedback is the statistical mean of the channel $-\mathbf{F}=$ $\mathrm{E}[\mathbf{H}]$ and $\mathcal{F}=\mathcal{C}^{M}$. Since the channel is isotropic, it is easy to see that Condition 2 will always be satisfied for the mean feedback case. As for condition 1, it can be simplified to

$$
\mathrm{E}_{\omega}\left[\frac{1}{1+P|\omega|^{2}}\right] \leq \frac{1}{1+P \alpha},
$$

where $\omega$ is distributed as $\mathcal{C N}(\|\mu\|, \alpha)$. Beamforming for the mean feedback channel is optimal if equation (13) is satisfied.

\section{F. Covariance Feedback}

In some scenarios, the channel may be varying too rapidly for the receiver to feedback even an estimate of the channel. In such cases, mean of the channel in such cases is assumed to be a zero vector and the covariance matrix can be calculated and fed back to the transmitter. For the covariance feedback scheme, $\mathbf{F}=\boldsymbol{\Sigma}$. Without any loss of generality, we assume that the input covariance matrix is diagonal and that the principal eigenvector is $\mathbf{q}=\left[\begin{array}{llll}1 & 0 & \cdots & 0\end{array}\right]^{\dagger}$. Since the channel is isotropic, condition 2 will be satisfied for all $\mathbf{H}$. Also note that since the covariance matrix is diagonal, the eigenvectors are along the $M$ dimensions. We then have $H_{\|}=H_{1}$ and $H_{\perp k}=H_{k} \quad \forall k \geq 2$. From the definition of the input covariance matrix, we have $\mathrm{E}\left[\left\|H_{k}\right\|^{2}\right]=\lambda_{k}$. Moreover $H_{j}$ and $H_{k}$ are independent for $j \neq k$. Condition 1 can then be written as

$$
\mathrm{E}_{\xi}\left[\frac{1}{1+P \lambda_{1} \xi}\right] \leq \frac{1}{1+P \lambda_{k}}, \quad 2 \leq k \leq M,
$$

where $\xi$ is an unit mean exponentially distributed variable. Therefore equation (14) is the necessary and sufficient condition for the optimality of beamforming for covariance information feedback.

\section{NUMERICAL RESULTS}

We now compare quantized direction feedback and mean feedback in an effort to determine how quantized direction feedback compares with other similar partial feedback schemes. To analyze them on the same platform, the mean information sent in the mean feedback case is also quantized. For the mean feedback case the channel is quantized using $N=2^{B}$ quantization vectors and the $B$ bit index of the closest quantization vector is fed back to the transmitter once every $D$ (delay) channel uses. For the quantized direction feedback case, the channel is quantized with $B / D$ bits $\left(N^{\frac{1}{D}}\right.$ quantization vectors) and fed back to the transmitter every channel use. Therefore, on the average both the feedback strategies receive the same amount of feedback $\left(B=\log _{2}(N)\right.$ bits). We consider an autoregressive model for the channel, so that

$$
\mathbf{H}(t)=a \mathbf{H}(t-1)+\sigma_{\mathbf{w}} \mathbf{w},
$$

where $\mathbf{w} \sim \mathcal{N}\left(\mathbf{0}, \frac{1}{M} \mathbf{I}\right), a,(0 \leq a \leq 1)$ is the forgetting factor and $\sigma_{\mathbf{w}}=\sqrt{\left(1-a^{2}\right)}$. We plot the average throughput for different values of the forgetting factor $a$ and the delay $D$.
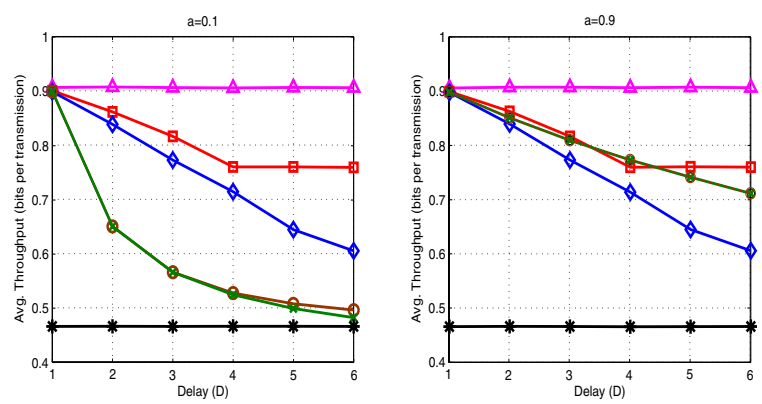

Fig. 2. Average throughput (in bits per transmission) of the perfect feedback bound $(\triangle)$, quantized direction feedback upper bound $(\square)$, quantized direction feedback achievable lower bound $(\diamond)$, mean feedback optimal capacity (o), mean feedback beamforming capacity $(\times)$ and no-feedback capacity $(*)$ for different values of the forgetting factor $a$.

Figure 2 shows the average throughputs of the two schemes with increasing delay in feedback for $a=0.1$ and 0.9 . The number of transmit antennas considered is $M=3, B=6$ bits and the average receive $\mathrm{SNR}$ is assumed to be $5 \mathrm{~dB}$. The throughput plots for perfect feedback and no-feedback are also shown for comparison. For simplicity, we assume that the input, channel and the output are real valued. 
When $D=1$ (channel feedback sent every channel change), for all $a$, both the schemes receive $B=6$ bits every channel use and consequently both the schemes have the same average throughput. Due to the large number of quantization vectors $\left(N=2^{6}=64\right)$, the achievable throughput performance is very close to that with perfect feedback. As $D$ increases, the quantized mean conveys less precise information about the instantaneous state of the channel. Consequently, the performance of mean feedback deteriorates with an increase in $D$.

For large $a(a \approx 1)$ the channel does not change much and mean feedback receives 6 bits of channel information while quantized direction feedback receives $\frac{6}{D}$ bits of channel information. Mean feedback therefore performs better than quantized feedback for large $a$. As $a$ decreases, the quality of the feedback decreases and consequently the average throughput offered by mean feedback decreases. Note that a large $a$ (higher memory) can reduce the feedback to achieve the same throughput. For $a=0$, the channel changes every transmission and for a large value of $D$, the performance of mean feedback approaches that of the no-feedback case. The performance of quantized feedback does not depend on the value of $a$, and therefore its throughput is unchanged with an increase in $a$.

\section{CONCLUSIONS}

We investigate the capacity of an general multiple transmit antenna channel with perfect channel knowledge at the receiver and partial/limited channel information at the transmitter. We derive a necessary and sufficient condition for beamforming to be capacity achieving in such systems. Based on this general condition, a set of simplified conditions are presented to help in determining the optimality of beamforming. These conditions are universal - applicable to all partial/limited feedback schemes in all ergodic fading channel conditions irrespective of the number of transmit antennas or transmit power. We demonstrate that the conditions can be used to derive previous results on the optimality of beamforming for several different partial/limited feedback schemes. The conditions can also be used to determine if beamforming is optimal in cases where optimality results are unknown. We demonstrate this with the quantized mean feedback scenario. An extension of the universal optimality condition to MIMO systems is provided in [25]. With Monte Carlo simulations, we also provide some numerical results comparing quantized feedback and mean feedback.

\section{REFERENCES}

[1] E. Telatar, "Capacity of Multi-antenna Gaussian Channels," European Transactions on Telecom, vol. 10, pp. 585-596, November 1999.

[2] E. Visotsky and U. Madhow, "Space-Time Transmit Precoding with Imperfect Feedback," IEEE Transactions on Information Theory, vol. 47, pp. 2632-2639, September 2001.

[3] Syed Ali Jafar and A. J. Goldsmith, " On Optimality of Beamforming for Multiple Antenna systems with Imperfect Feedback," IEEE International Symposium on Information Theory, p. 321, June 2001.

[4] Syed Ali Jafar and A. J. Goldsmith, "Transmitter Optimization and Optimality of Beamforming for Multiple Antenna Systems with Imperfect Feedback," IEEE Transactions on Wireless Communications, vol. 3, pp. 1165-1175, July 2004.
[5] S. Simon and A. Moustakas, "Optimality of Beamforming in Multiple Transmitter Multiple Receiver Communication Systems with Partial Channel Knowledge," DIMACS Workshop on Signal Processing for Wireless Communications, October 2002.

[6] A. Moustakas and S. Simon, "Optimizing Multi-Transmitter SingleReceiver (MISO) Antenna Systems with Partial Channel Knowledge," IEEE Transactions on Information Theory, pp. 2770-2780, October 2003.

[7] Y. Xie, C. Georghiades, and A. Arapostathis, "Minimum Outage Probability Transmission With Imperfect Feedback for MISO Fading Channels," IEEE Transactions on Wireless Communications, vol. 4, pp. 1084 1091, May 2005.

[8] E. Jorswieck and H. Boche, "Channel Capacity and Capacity-Range of Beamforming in MIMO Wireless Systems under Correlated Fading with Covariance Feedback," IEEE Transactions on Wireless Communications, vol. 3, pp. 1543- 1553, September 2004.

[9] Syed Ali Jafar and A. J. Goldsmith, "Isotropic Fading Vector Broadcast Channels: The Scalar Upperbound and Loss in Degrees of Freedom," IEEE Transactions on Information Theory, vol. 51, pp. 848-857, March 2005.

[10] Sudhir Srinivasa and Syed Ali Jafar, "Capacity of the Isotropic Fading Multiple Antenna Downlink with Magnitude Feedback," IEEE Vehicular Technology Conference, September 2004.

[11] K.K. Mukkavilli and A. Sabharwal and E. Erkip and B. Aazhang, "On Beamforming with Finite Rate Feedback in Multiple Antenna Systems," IEEE Transactions on Information Theory, vol. 49, pp. 2562-2579, October 2003

[12] D. J. Love and R. W. Heath Jr. and T. Strohmer, "Grassmannian Beamforming for Multiple-Input Multiple-Output Wireless Systems," IEEE International Conference on Communications, vol. 4, pp. 26182622, May 2003.

[13] D. J. Love and R. W. Heath Jr. and T. Strohmer, "Grassmannian Beamforming for Multiple-Input Multiple-Output Wireless Systems," IEEE Transactions on Information Theory, vol. 49, pp. 2735-2747, October 2003.

[14] D. J. Love and R. W. Heath Jr., "Grassmannian Beamforming on Correlated MIMO Channels," IEEE International Conference on Communications, vol. 1, pp. 106-110, November - December 2004.

[15] P. Xia and S. Zhou and G. B. Giannakis, "Design and Analysis of Transmit Beamforming based on Limited-Rate Feedback," IEEE Transactions on Information Theory, 2005.

[16] P. Xia and S. Zhou and G. B. Giannakis, "Achieving the Welch Bound with Difference Sets," IEEE Transactions on Information Theory, vol. 51, May 2005.

[17] Syed Ali Jafar and Sudhir Srinivasa, "Capacity of the Isotropic Fading Vector Channel with Quantized Channel Direction Feedback," Asilomar Conference on Signals, Systems and Computers, November 2004.

[18] Sudhir Srinivasa and Syed Ali Jafar, "Vector Channel Capacity with Quantized Feedback," IEEE International Conference on Communications, May 2005.

[19] Syed Ali Jafar and Sudhir Srinivasa, "On the Optimality of Beamforming with Quantized Feedback," Submitted to IEEE Transactions on Communications.

[20] Sudhir Srinivasa and Syed Ali Jafar and Sriram Vishwanath, "Does Beamforming achieve Outage Capacity with Direction Feedback?," Communication Theory Workshop, June 2005.

[21] June Chul Roh and Bhaskar D. Rao, "Performance Analysis of Multiple Antenna Systems with VQ-Based Feedback," Asilomar Conference on Signals, Systems and Computers, November 2004.

[22] June Chul Roh and Bhaskar D. Rao, "Channel Feedback Quantization Methods for MISO and MIMO Systems," IEEE International Symposium on Personal, Indoor and Mobile Radio Communications, September 2004.

[23] June Chul Roh and Bhaskar D. Rao, "Design and Analysis of MIMO Spatial Multiplexing Systems with Quantized Feedback," Submitted to IEEE Transactions on Signal Processing.

[24] Syed Ali Jafar, S. Vishwanath and A. J. Goldsmith, "Channel Capacity and Beamforming for Multiple Transmit and Receive Antennas with Covariance Feedback," IEEE International Conference on Communications, 2001.

[25] Sudhir Srinivasa and Syed Ali Jafar, "The Optimality of Beamforming: A Unified View," In preparation. 